\title{
A case report of ultrasound-guided interstitial brachytherapy for abdominal wall metastases of ovarian cancer
}

\author{
Dan Shi, MD*, Ning Wu, MD*, Hongfu Zhao, MS, Mingyuan He, MD, Dongmei Han, BS, Guanghui Cheng, MD, PhD \\ *These two authors contributed equally to this work.
}

Department of Radiation Oncology. China-Japan Union Hospital of Jilin University, Changchun, China

\begin{abstract}
Purpose: To report the treatment effect of interstitial brachytherapy for abdominal wall metastases of ovarian cancer.

Material and methods: The patient is a 44-year-old female with a diagnosis of stage IA ovarian cancer. After surgery and two cycles of chemotherapy with paclitaxel and carboplatin, the patient noticed dull pain in the lower abdomen and found a mass located in the subcutaneous tissue, below the operative incision. A diagnostic biopsy showed abdominal wall metastases. After external radiotherapy with a dose of $39.6 \mathrm{~Gy}$ in 22 fractions, the residual tumor was treated with interstitial brachytherapy under ultrasound guidance. The brachytherapy dose was 18 Gy in 6 fractions of 3 Gy each.

Results: After 3 weeks of brachytherapy, the tumor had disappeared completely. Interstitial brachytherapy was feasible.

Conclusions: Interstitial brachytherapy may be a proposed treatment strategy for inoperable superficial metastases, especially for low radiosensitivity cancer.

J Contemp Brachytherapy 2015; 7, 1: 81-85 DOI: $10.5114 /$ jcb.2015.48574
\end{abstract}

Key words: abdominal wall metastases, interstitial brachytherapy, ovarian cancer.

\section{Purpose}

Ovarian cancer is one of the most common malignant tumors in females. Treatment involves surgery, chemotherapy, and sometimes radiotherapy [1]. Surgery, which is performed by a specialist in gynecologic oncology, is the preferred treatment method. For patients with advanced disease, a combination of surgical reduction and a chemotherapy regimen is standard [2]. Chemotherapy, which depends on the histology of the tumor, after surgery has been a general standard of care for ovarian cancer for decades [3]. External radiotherapy is not considered to be an effective treatment for ovarian cancer because of its low radiosensitivity. Brachytherapy with high dosage to target volume and restriction of normal tissue exposure may play a meaningful role in the treatment of ovarian cancer and its metastases. It provides advantages over external beam radiation therapy: the tumor can be treated with very high doses of localized radiation, while reducing the probability of unnecessary damage to surrounding healthy tissues [4,5]. In this context, we propose a technique using high-dose-rate (HDR) intersti- tial brachytherapy to treat abdominal wall metastases of ovarian cancer.

\section{Case description}

We encountered a 44-year-old patient with abdominal wall metastases from ovarian cancer complaining of pain in the lower abdomen. Six months before being admitted to our clinic, she was diagnosed with ovarian cancer (stage IA), and received radical surgery on March $18^{\text {th }}$ 2014. The pathology result after surgery was serous papillary adenocarcinoma of the left ovary with no lymphovascular, nerve or omentum involvement. Taking into account general state, pathological examination, stage of the disease, and medical history, the patient qualified for adjuvant chemotherapy. Chemotherapy with $175 \mathrm{mg} / \mathrm{m}^{2}$ of paclitaxel and $400 \mathrm{mg} / \mathrm{m}^{2}$ of carboplatin was immediately started and continued at the same dosage in the $2^{\text {nd }}$ cycle.

After 1 month, the patient noticed a dull pain in the lower abdomen. Ultrasound examination in June 2014 revealed a mass $\left(4.2 \times 2.7 \times 4.2 \mathrm{~cm}^{3}\right)$ located in the subcutaneous tissue of the lower abdomen, below the surgical 


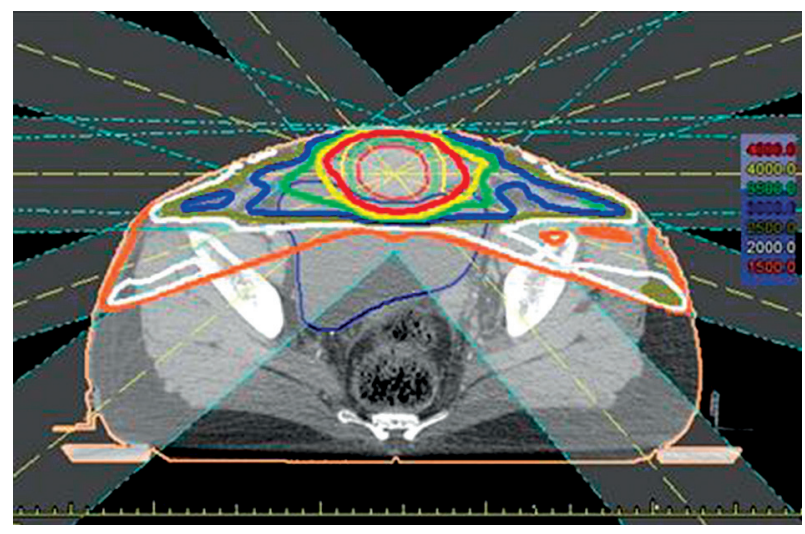

Fig. 1. Dose distribution in the external radiotherapy plan

incision. A diagnostic biopsy, prompted by immunohistochemical markers, revealed a metastatic malignant tumor (originating from the ovary). The proposal of surgery was rejected by patient. The patient therefore underwent initial radiotherapy by external beam: a total of 39.6 Gy in 1.8 Gy fractions for the tumor area (see Fig. 1). After external radiotherapy, the tumor size had decreased to 2.2 $\times 1.5 \times 2.5 \mathrm{~cm}^{3}$ according to the pelvic MRI examination. The symptoms in the patient's lower abdomen, however, were not markedly relieved.

High-dose-rate interstitial brachytherapy, following external radiotherapy within one week, with a total dose of $18 \mathrm{~Gy}$ in 6 fractions over 3 days (3 Gy each fraction, twice a day with a $6 \mathrm{~h}$ interval) was administered to the patient. OncoSmart ProGuide needles (Nucletron, an Elekta company, Elekta AB, Stockholm, Sweden) were used and the treatment was performed under anesthesia. Electrocardiogram, arterial oxygen pressure, respiration, and blood pressure monitoring were performed during the procedure. Four applicator needles $(1.1 \mathrm{~mm}$ in external diameter and $20 \mathrm{~cm}$ in length) were inserted into the target under ultrasound guidance, with an interval of $1 \mathrm{~cm}$ to ensure adequate dose distribution and target volume coverage (see Fig. 2 and 3). OncoSmart ProGuide CT-Markers were put inside the catheters in order to facilitate their reconstruction. After implanting the applicators, fine-pitch $(2 \mathrm{~mm}) \mathrm{X}$-ray CT images were acquired and transferred to the treatment planning computer (see Fig. 4). The CT-imaging data combined with the MR before interstitial brachytherapy were used to contour the gross target volume (GTV) and the clinical target volume (CTV). The CTV was expanded from GTV by $2.0 \mathrm{~cm}$ and restricted by the volume of the critical organs (especially the small intestine) (see Fig. 5). A CT-based treatment plan was created using a graphic optimization tool (Treatment Planning System Oncentra V4.3, Nucletron, an Elekta company, Elekta AB, Stockholm, Sweden). The normalization and optimization to the target volume was performed.

Dose volume histogram (DVH) parameters are recommended for the evaluation of target volume and organs at risk (OARs). The dose distribution to target volume and
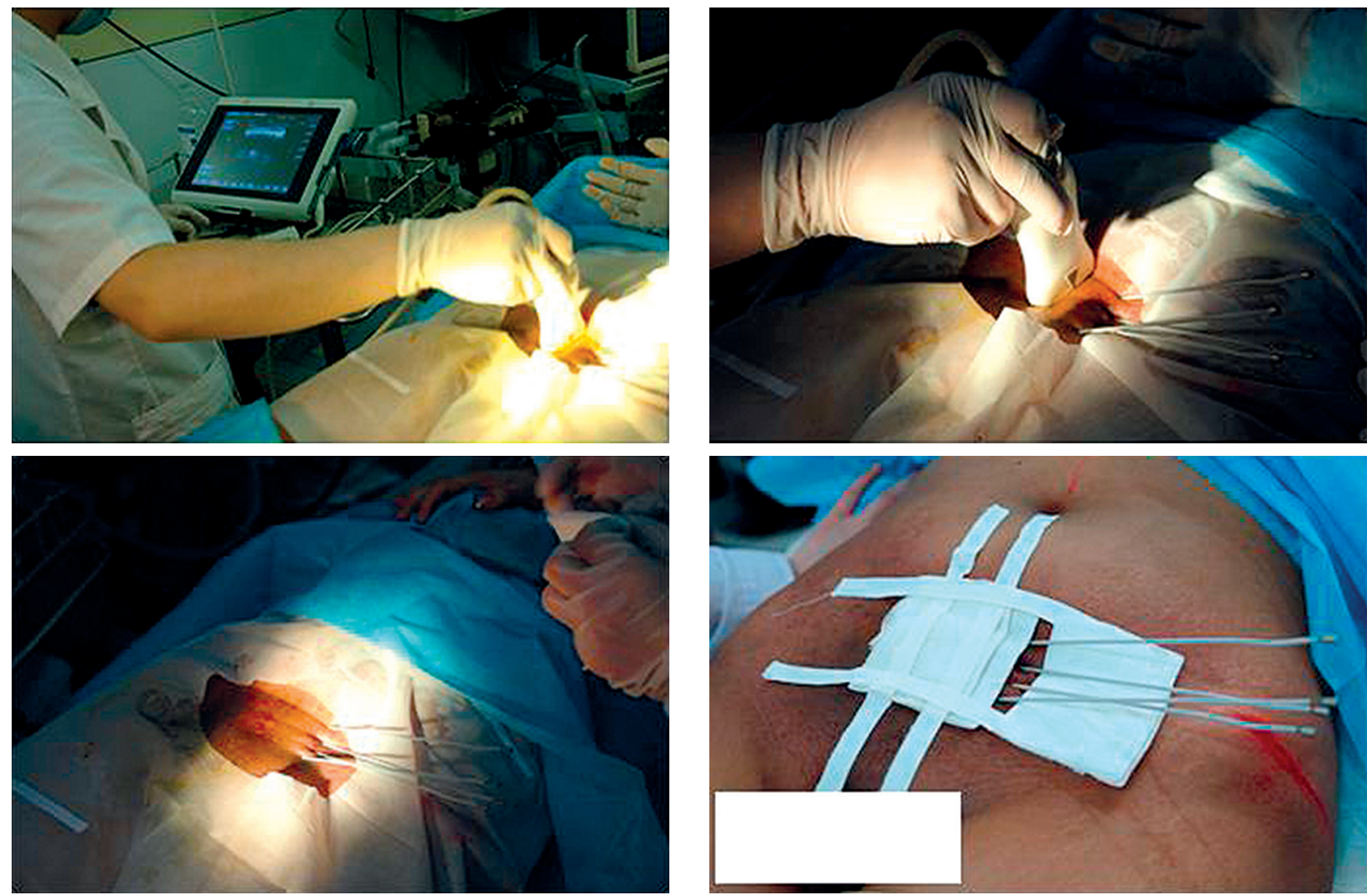

Fig. 2. Four applicator needles were inserted into the target under ultrasound guidance 

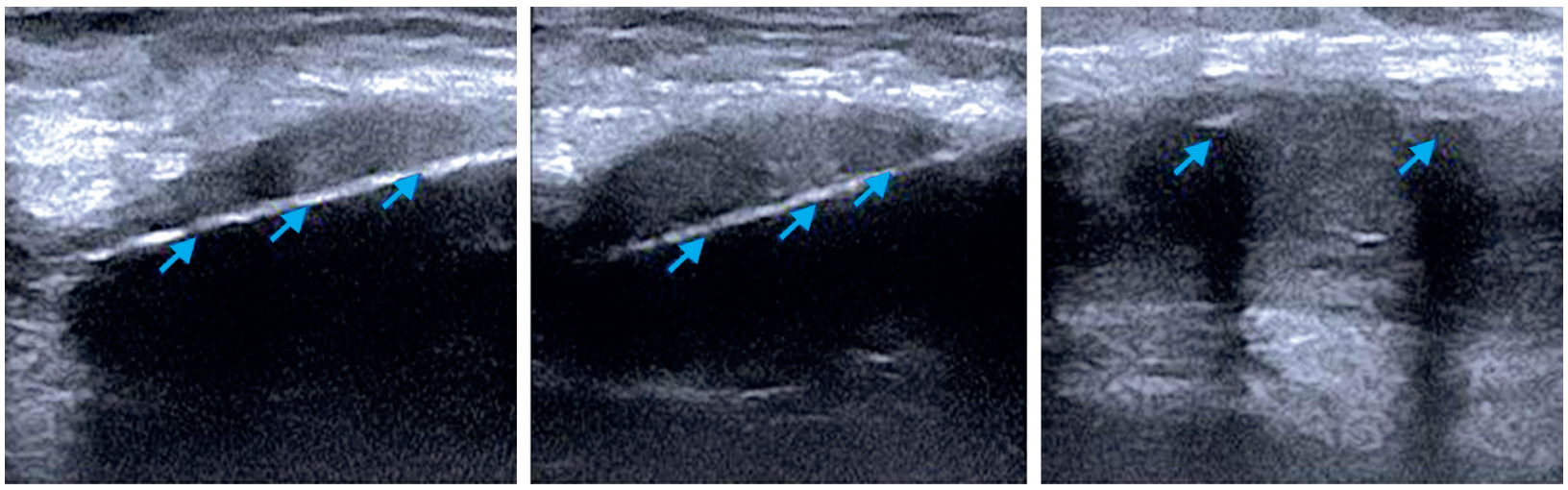

Fig. 3. The ultrasound images of inserting applicator needles
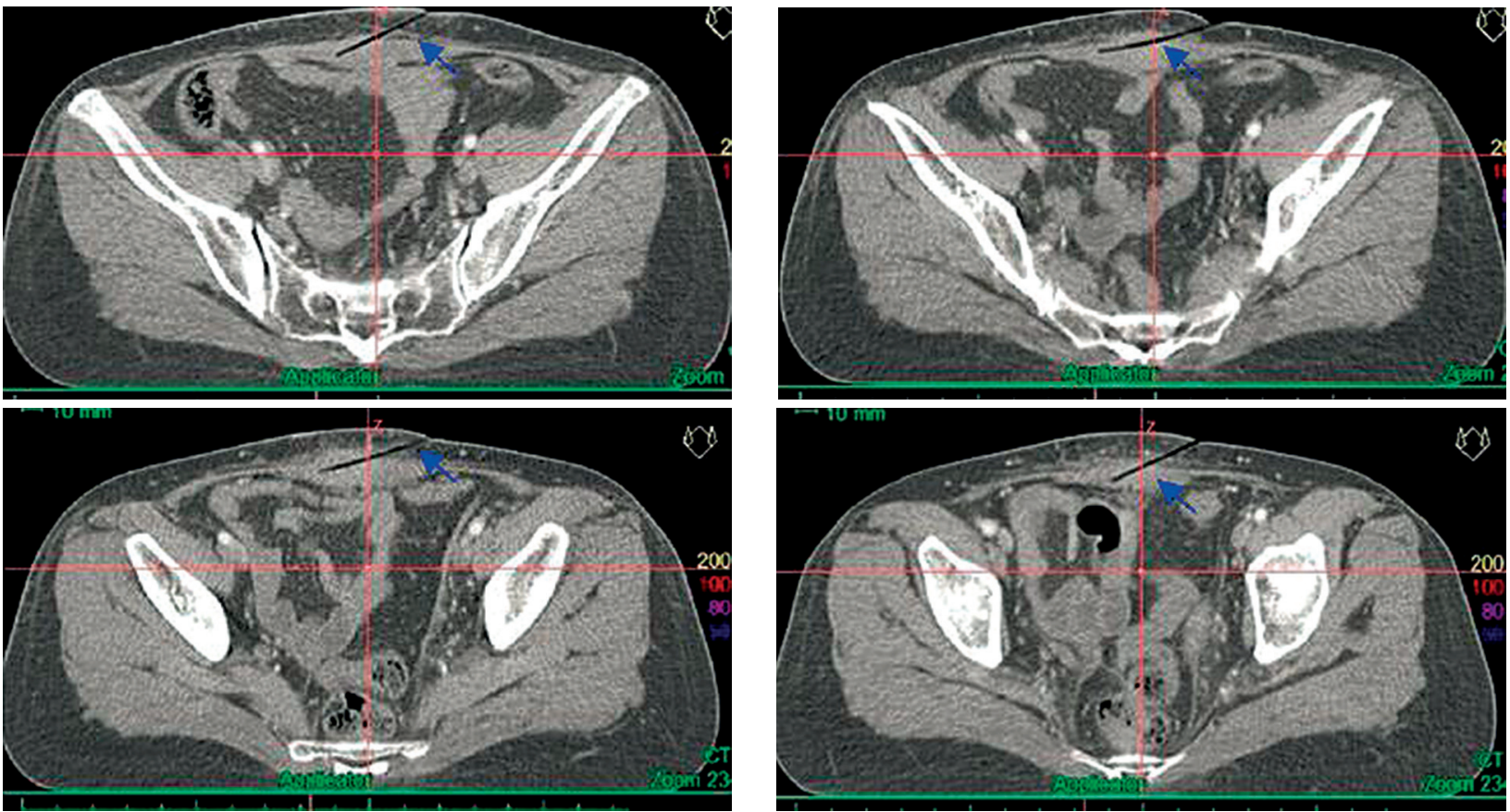

Fig. 4. The position of each applicator needle on CT images

OARs are shown in Figure 6A. In the brachytherapy plan, 18 Gy was prescribed to $100 \%$ of the target volume, and $\mathrm{D}_{2 \mathrm{cc}}$ (minimum dose to the most irradiated volume of $2 \mathrm{ml}$ ) of the small intestine was mainly considered. In the external radiotherapy plan, 39.6 Gy in 1.8 Gy per fraction was prescribed to the target. The equivalent dose for a 2 Gy fraction schedule was calculated using the EQD2 model, at $\alpha / \beta=3\left(\mathrm{~Gy}_{\mathrm{EQD} 2, \alpha / \beta=3}\right)$ for the small intestine and $\alpha / \beta=10\left(\mathrm{~Gy}_{\mathrm{EQD} 2, \alpha / \beta=10}\right)$ for the target $[6,7]$. Dose volume histogram parameters were analyzed taking into account the volume ratio of the target receiving $90 \%$ or $150 \%$ of the prescription dose $\left(\mathrm{V}_{90 \%}\left[\mathrm{~V}_{2.7 \mathrm{~Gy}}\right]\right.$ and $\mathrm{V}_{150 \%}$ $\left[\mathrm{V}_{4.5 \mathrm{~Gy}}\right]$, respectively), the equivalent dose delivered to $98 \%$ and $50 \%$ of the clinical target volume $\left(\mathrm{D}_{98 \%}\right.$ and $\mathrm{D}_{50 \%}$ respectively), and the volume ratio of the small intestine receiving $25 \mathrm{~Gy}, 35 \mathrm{~Gy}$ and $45 \mathrm{~Gy}$ of the equivalent dose $\left(\mathrm{V}\left(25 \mathrm{~Gy}_{\mathrm{EQD} 2}\right), \mathrm{V}\left(35 \mathrm{~Gy}_{\mathrm{EQD} 2}\right)\right.$ and $\mathrm{V}\left(45 \mathrm{~Gy}_{\mathrm{EQD} 2}\right)$ respec- tively). The following values were accepted: below $\mathrm{V}_{90 \%}$ was $5.6 \%, \mathrm{~V}_{150 \%}$ was $63.2 \%$; $\mathrm{D}_{98 \%}$ was $54.5 \mathrm{~Gy}, \mathrm{D}_{50 \%}$ was $120.4 \mathrm{~Gy} ; \mathrm{V}\left(25 \mathrm{~Gy}_{\mathrm{EOD} 2}\right)$ was $0.9 \%, \mathrm{~V}\left(35 \mathrm{~Gy}_{\mathrm{EOD} 2}\right)$ was $1.1 \%$, $\mathrm{V}\left(45 \mathrm{~Gy}_{\mathrm{EQD} 2}\right)$ was $1.3 \% ; \mathrm{D}_{0.1 \mathrm{cc}}$ of the small intestine was 33.6 $\mathrm{Gy}_{\mathrm{EQD} 2, \alpha / \beta=3}, \mathrm{D}_{1 \mathrm{cc}}$ was $24.7 \mathrm{~Gy}_{\mathrm{EQD} 2, \alpha / \beta=3}$ and $\mathrm{D}_{2 \mathrm{cc}}$ was $21.3 \mathrm{~Gy}_{\mathrm{EQD} 2, \alpha / \beta=3}$.

After transporting the planning data to an iridium-192 remote afterloader system (Microselectron HDR Ir-192, Nucletron, an Elekta company, Elekta AB, Stockholm, Sweden), irradiation was started. The irradiation took approximately $5 \mathrm{~min}$, as shown in Figure 6 (B, C and D). The needles were removed after irradiation was complete, and the patient was discharged after $2 \mathrm{~h}$ under observation. No complications were reported during the treatment and the brachytherapy was well tolerated by the patient. The patient is regularly followed up at our affiliated clinics. 


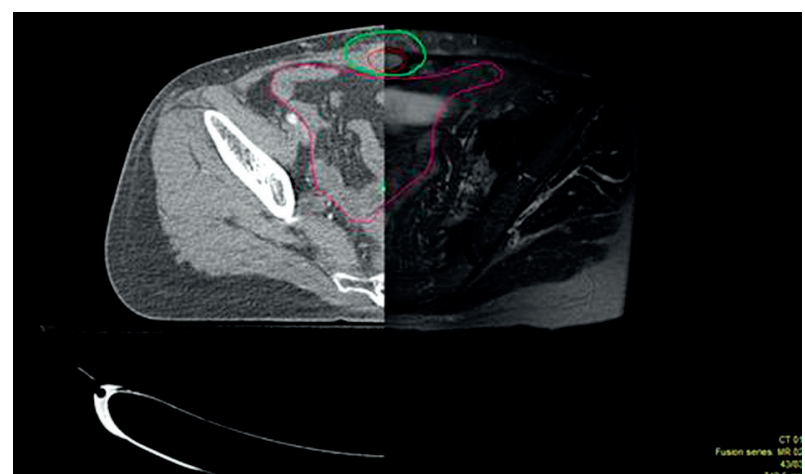

Fig. 5. The target contour of interstitial brachytherapy based $\mathrm{CT}$ and MR fusion

\section{Results}

During the follow-up examination in the $3^{\text {rd }}$ week after brachytherapy, remission of the metastatic cancer was observed. The abdominal wall mass had disappeared according to the MR results (see Fig. 7). During the follow-up visit (2 months after therapy), only radiation-induced skin injury (RTOG, Grade 1) was observed. According to the recent follow-up over 5 months after therapy, there were no signs or symptoms of abdominal complications and no evidence of recurrence at the site of re-irradiation. Chemotherapy will be continued depending on the personal tolerance and general patient condition.

\section{A}
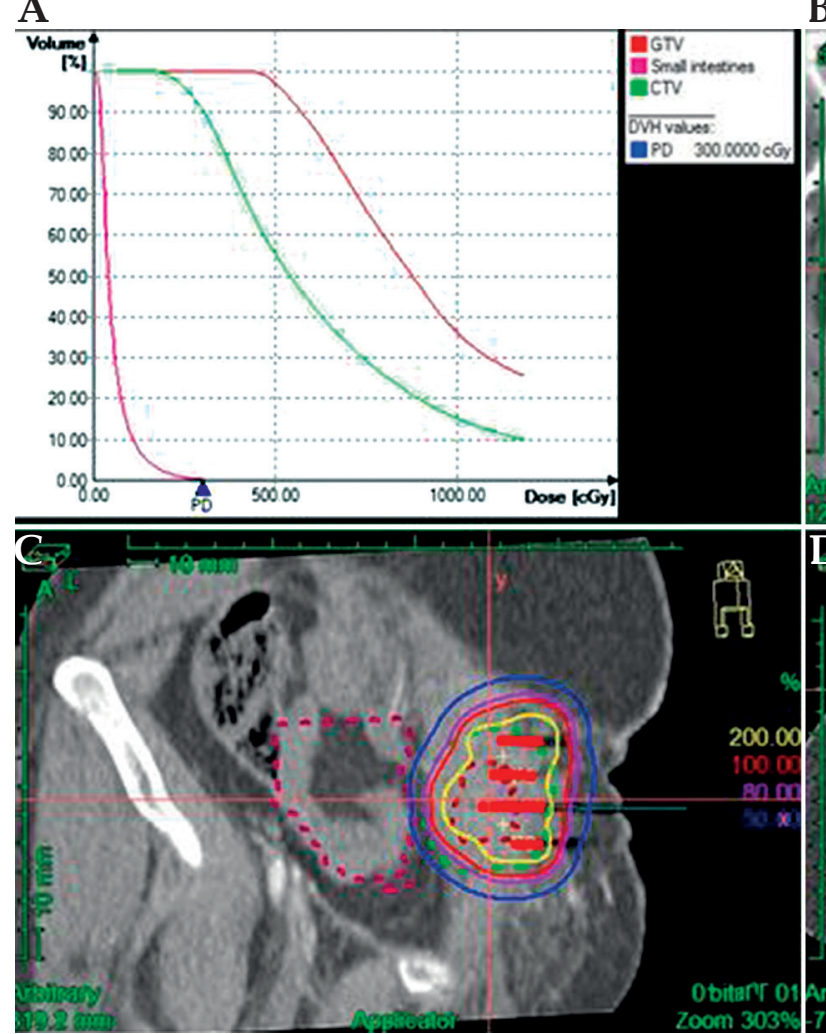

\section{Discussion}

Abdominal wall metastases from ovarian cancer is not a common clinical situation. Generally, external radiotherapy is not a conventional treatment for ovarian cancer patients after surgery, because of the lower radiosensitivity of this tumor. It is rare to see abdominal wall metastases present at such an early stage as in the present case. However, information on previous studies on the use of interstitial brachytherapy for ovarian cancer patients has not been reported. Kishi et al. [8], administered interstitial brachytherapy for abdominal wall metastases of colorectal cancer. Although the common pathology result for colorectal cancer is adenocarcinoma and its radiosensitivity is not high, the effect after the interstitial brachytherapy was acceptable. In order to control the residual tumor, interstitial brachytherapy was used after external beam therapy for this patient. Four applicator needles were inserted with the guidance of ultrasound. The DVH showed that GTV was covered with high dose irradiation but the dosage to the small intestines was relative low. All the tumor had disappeared 3 weeks later without any serious complications. HDR interstitial brachytherapy provided a superior therapeutic ratio compared with external radiotherapy, and enabled curative dose treatment with prominent therapeutic enhancement.

\section{Conclusions}

Interstitial brachytherapy is a safe and practical supplementary method on top of external beam radiothera-

B
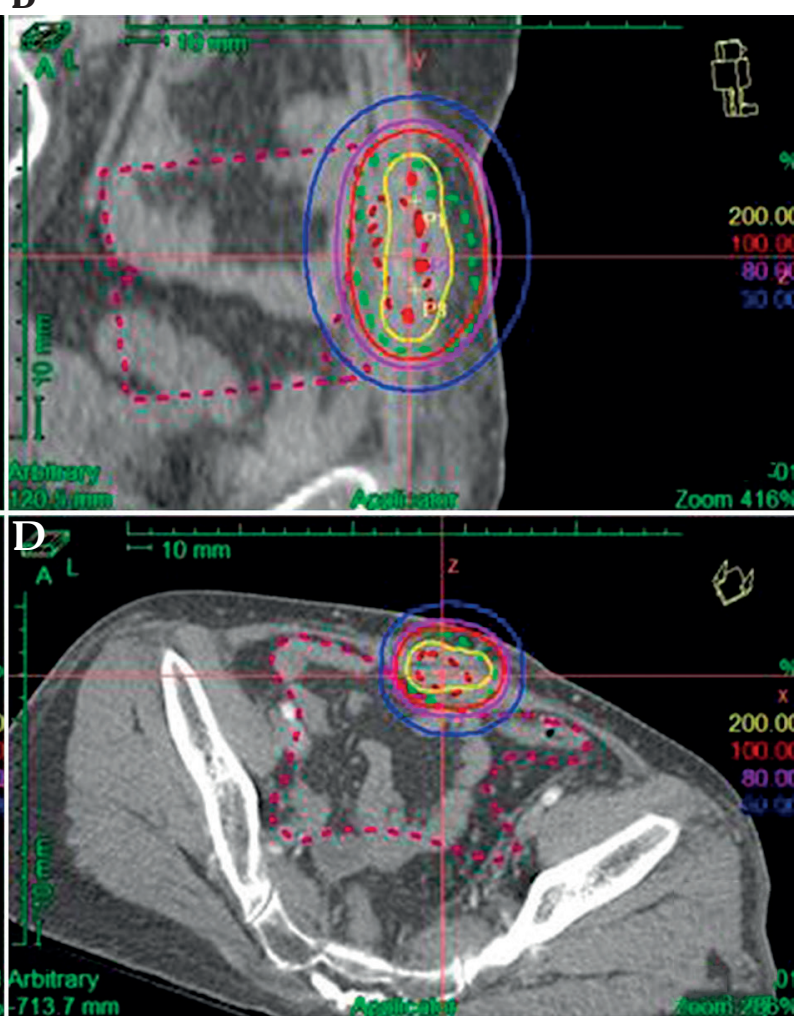

Fig. 6. A) The dose volume histogram (DVH) parameters. It shows the high dose distribution of GTV and CTV and low dose distribution of small intestines. B-D) The dose distribution of horizontal, sagittal + coronal positions. The red line shows the position of $\mathrm{V}_{100}$ covering GTV completely. Just a small part of $\mathrm{V}_{50}$ (showed by blue line) covered the small intestines 

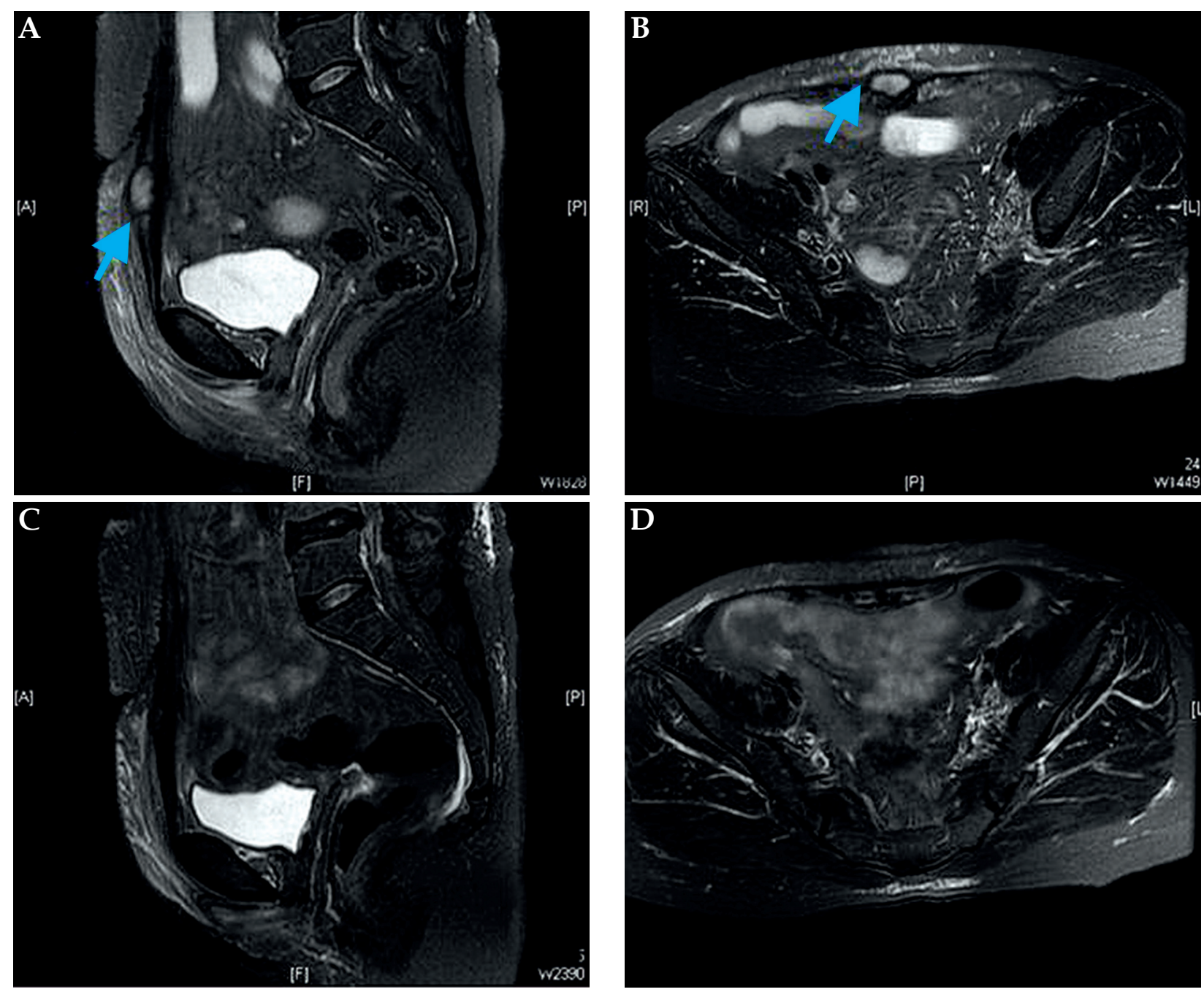

Fig. 7. The MR of abdominal wall (A, B) before, and (C, D) 3 weeks after brachytherapy

py. It is well tolerated by patients because of the high dose delivered to the tumor and the simultaneous effective protection to the normal organs. Interstitial brachytherapy may be a proposed scheme to treat inoperable superficial metastases, especially for low radiosensitivity cancer. It is important to pay careful attention to critical organ protection during implementation of interstitial brachytherapy and an individual approach is needed in every patient.

\section{Acknowledgements}

This study was partially supported by a grant from the National Natural Science Foundation of China (81201737) and Bethune Medical Research Program (201310724).

\section{Disclosure}

Authors report no conflict of interest.

\section{References}

1. Chobanian N, Dietrich CS. Ovarian cancer. Surg Clin North Am 2008; 88: 285-299.
2. Ehrlich PF, Teitelbaum DH, Hirschi RB et al. Excision of large cystic ovarian tumors: combining minimal invasive surgery techniques and cancer surgery - the best of both worlds. J Pediatr Surg 2007; 42: 890-893.

3. McGuire WP, Markman M. Primary ovarian cancer chemotherapy: current standards of care. Br J Cancer 2003; 89 Suppl 3: S3-S8.

4. Gerbaulet A, Pötter R, Mazeron J et al. The GEC ESTRO handbook of brachytherapy. ACCO, Leuven 2005.

5. Nag S. High dose rate brachytherapy: its clinical applications and treatment guidelines. Technol Cancer Res Treat 2004; 3: 269-287.

6. Kirisits C, Potter R, Lang S et al. Dose and volume parameters for MRI-based treatment planning in intracavitary brachytherapy for cervical cancer. Int J Radiat Oncol Biol Phys 2005; 62: 901-911.

7. Lang S, Kirisites C, Dimopoulus J et al. Treatment planning for MRI assisted brachytherapy of gynecologic malignancies based on total dose constraints. Int J Radiat Oncol Biol Phys 2007; 69: 619-627.

8. Kishi K, Takifuji K, Shirai S et al. Brachytherapy technique for abdominal wall metastases of colorectal cancer: ultrasound-guided insertion of applicator needle and a skin preservation method. Acta Radiol 2006; 47: 157-161. 